\title{
Efficient synthesis of piperazinyl amides of 18ß-glycyrrhetinic acid
}

\author{
Dong Cai ${ }^{1}$, ZhiHua Zhang ${ }^{2}$, Yufan Meng ${ }^{3}$, KaiLi Zhu ${ }^{3}$, LiYi Chen ${ }^{3}$, ChangXiang Yu ${ }^{3}$, \\ ChangWei $\mathrm{Yu}^{3}$, ZiYi Fu${ }^{3}$, DianShen Yang ${ }^{*}$ and YiXia Gong ${ }^{*} 1,4$
}

\author{
Full Research Paper \\ Address: \\ ${ }^{1}$ College of Public Basic Sciences, Jinzhou Medical University, \\ Jinzhou, 121001, China, ${ }^{2}$ School of Chemical and Environmental \\ Engineering, Liaoning University of Technology, Jinzhou, 121001, \\ China, ${ }^{3}$ College of Pharmacy, Jinzhou Medical University, Jinzhou, \\ 121001, China and ${ }^{4}$ College of Pharmacy, Jiamusi University, \\ Jiamusi, 154007, China \\ Email: \\ DianShen Yang * - 86384370@qq.com; YiXia Gong* - \\ gongyixia_2006@163.com \\ * Corresponding author \\ Keywords: \\ 18ß-glycyrrhetinic acid; piperazinyl amides; synthesis \\ Beilstein J. Org. Chem. 2020, 16, 798-808. \\ doi:10.3762/bjoc. 16.73 \\ Received: 16 November 2019 \\ Accepted: 09 April 2020 \\ Published: 21 April 2020 \\ Associate Editor: S. Bräse \\ (c) 2020 Cai et al.; licensee Beilstein-Institut. \\ License and terms: see end of document.
}

Open Access

\begin{abstract}
In the present study, a practical method to prepare piperazinyl amides of $18 \beta$-glycyrrhetinic acid was developed. Two main procedures for the construction of important intermediate $\mathbf{8}$ are discussed. One procedure involves the amidation of 1-Boc-piperazine

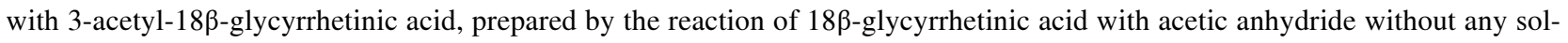
vent at $130{ }^{\circ} \mathrm{C}$. The other procedure to prepare compound $\mathbf{8}$ involves the amidation of $18 \beta$-glycyrrhetinic acid followed by the esterification with acetic anhydride. Finally, compound $\mathbf{8}$ underwent $N$-Boc deprotection to prepare product $\mathbf{4}$. To ascertain the scope of the reaction, another $\mathrm{C}-3$ ester derivative $\mathbf{1 7}$ was tested under the optimized reaction conditions. Furthermore, the reasons for the appearance of byproducts were elucidated. Crystallographic data of a selected piperazinyl amide is reported.
\end{abstract}

\section{Introduction}

Glycyrrhizin was the major bioactive component in Glycyrrhiza uralensis fisch root. $18 \beta$-glycyrrhetinic acid (1, Figure 1) was then obtained by hydrolysis of glycyrrhizin. 18ß-glycyrrhetinic acid and its derivatives have been extensively investigated in medicinal chemistry for their various biological activities, including anti-inflammatory [1], antiulcer [2], antioxidative [3], antitumor [4], and antibacterial [5] activity and their proberties as hepatic protective agents [6].
Over the past few decades, the emphasis was placed primarily on explaining structure-activity relationships of $18 \beta$-glycyrrhetinic acid derivatives to enhance their biological activity. The structure of $18 \beta$-glycyrrhetinic acid offered numerous possibilities to chemical modifications, thereby leading to the formation of novel derivatives [7-9]. There are three functional groups at C-3, C-11 and C-30 in the structure of $18 \beta$-glycyrrhetinic acid amenable to chemical modifications. 


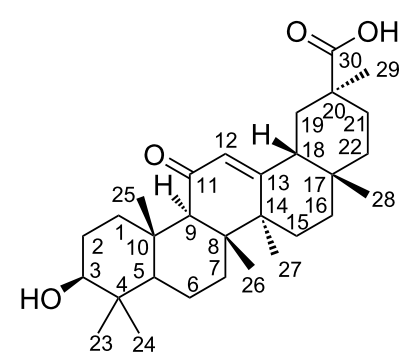

1

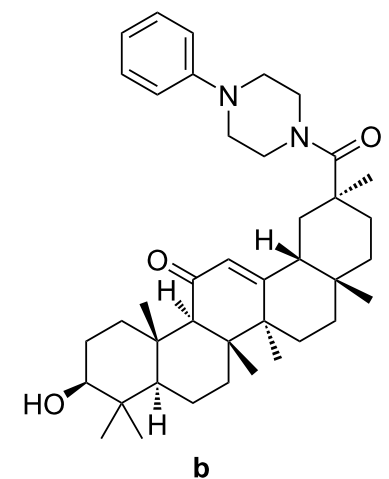<smiles>CC1(C)C2CC[C@]3(C)C(C(=O)C=C4[C@@H]5C[C@](C)(C(=O)O)CC[C@]5(C)CC[C@]43C)[C@@]2(C)CC[C@H]1OC(=O)c1cccc(C(=O)O)c1</smiles>

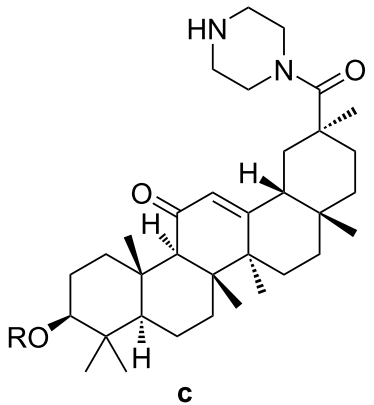

Figure 1: Chemical structure of 18ß-glycyrrhetinic acid and known derivatives.

The modifications at the $\mathrm{C} 3-\mathrm{OH}$ group of $18 \beta$-glycyrrhetinic acid are identified to be relatively common and effective. The modification of the $\mathrm{C} 3-\mathrm{OH}$ group, altering the molecular polarity of $18 \beta$-glycyrrhetinic acid, may be an advantage in achieving better cytotoxicity or antiproliferative activity [8-10]. For instance, the hydroxy group can be converted into an oxime, acyloxyimino, alkoxyimino, alkoxy and 3-oxo group [9]. As a proteasome inhibitor, compound a suppresses the chymotrypsin-like activity of the proteasome in MT4 cells with an $\mathrm{IC}_{50}$ of $0.22 \mu \mathrm{M}$, nearly 100 -fold more potent than $18 \beta$ glycyrrhetinic acid.

Besides, the C-30 carboxyl group is often esterified or amidated in order to enhance the antitumor or other efficacy of $18 \beta$ glycyrrhetinic acid derivatives [11]. A novel piperazinyl amide b exhibits the optimal inhibitory activity against MCF-7 and can be further developed as a potent VEGFR2 and antitumor agent [12].

Piperazinyl amide fragments have the ability to form several hydrogen bonds, modulate the acid-base equilibrium constant and change the octanol-water partition coefficient [13]. They are considered as the basic motif for designing many biologically active molecules $[14,15]$. Some piperazinyl amides of $18 \beta$ glycyrrhetinic acid (c) are synthesized using various methods. A method involves the C30-position of the acyl chloride with symmetric piperazine $[16,17]$. In this case, the acyl chloride can be prepared without purification, and the total yield over two steps can reach $81 \%$ [16]. Such structures (c) can also be formed by treating the $\mathrm{C} 30$ carboxyl group with piperazine in the presence of activators (e.g., 1-ethyl-3-(dimethylaminopropyl)carbodiimide hydrochloride (EDCl), 1-hydroxybenzotriazole (HOBt) and trimethylamine [18]), the yield of piperazinyl amide derivatives was $80.6 \%$ [19]. These two methods are complicated through the side formation of bisamide, obviously, due to the competitive attack on the $\mathrm{N}$ atoms of the symmetric diamine. An alternative method [12] to prepare such piperazinyl amides (c) involves the amidation of $18 \beta$-glycyrrhetinic acid with 4-substituted phenylpiperazines in the presence of $N, N$-dicyclohexylcarbodiimide (DDC) and HOBt. Under such reaction conditions, the bisamide as byproduct can be evitable, whereas the substituted piperazine compounds will not be readily available.

In principle, an efficient approach to the synthesis of structural analogues of (c) is based on the reaction of carboxylic acid with $\mathrm{N}$-Boc-protected aliphatic diamine in the presence of activators and then deprotection of the tert-butoxycarbonyl (Boc) group using trifluoroacetic acid (TFA) [20]. Compared with the mentioned methods, the latter method achieves the higher yield (up to $90 \%$ ) without the formation of byproducts [21].

Given the flexible 18 $\beta$-glycyrrhetinic acid scaffold in the design of biologically active compounds, a novel and efficient method 
was reported to prepare $\mathrm{C} 3$ ester derivatives or/and C30 piperazinyl amides (c).

$18 \beta$-Glycyrrhetinic acid belongs to the class of ursane-type pentacyclic triterpenoids, and the synthetic methods studied here can also apply to the modification of structurally similar other triterpenoic acids, but further experimental verification is needed.

\section{Results and Discussion}

The synthetic route to $18 \beta$-glycyrrhetinic acid piperazinyl amide 4 was originally reported by Sommerwerk [16] (Scheme 1). In this case, 18 $\beta$-glycyrrhetinic acid reacted with acetic anhydride in the presence of triethylamine to give 3-acetyl-18ß-glycyrrhetinic acid (2), which by successive chlo- rination with oxalyl chloride yielded acyl chloride 3 . Without isolation, the intermediate 3 reacted with piperazine to give $18 \beta$-glycyrrhetinic acid piperazinyl amide 4 . The total yield of chlorination and amidation reactions was $67 \%$.

In the beginning, the reaction of acyl chloride 3 with piperazine was taken as a prototypical case to ascertain and screen the experimental conditions. First, the solution with intermediate 3 was cooled to $0{ }^{\circ} \mathrm{C}$, and triethylamine (1.1 equiv) as well as piperazine (3.0 equiv) were added. After $30 \mathrm{~min}$ of stirring, the solvent was removed in vacuo and the residue was purified by column chromatography to afford amide $\mathbf{4}$ and bisamide 5 . When increasing the amount of piperazine, relatively high yields of amide 4 would be achieved (Table 1, entries 1-3). Given the high reactivity of the acyl chloride with piperazine,

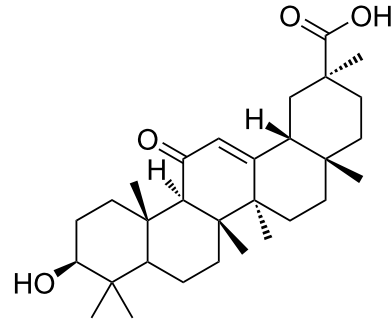

1

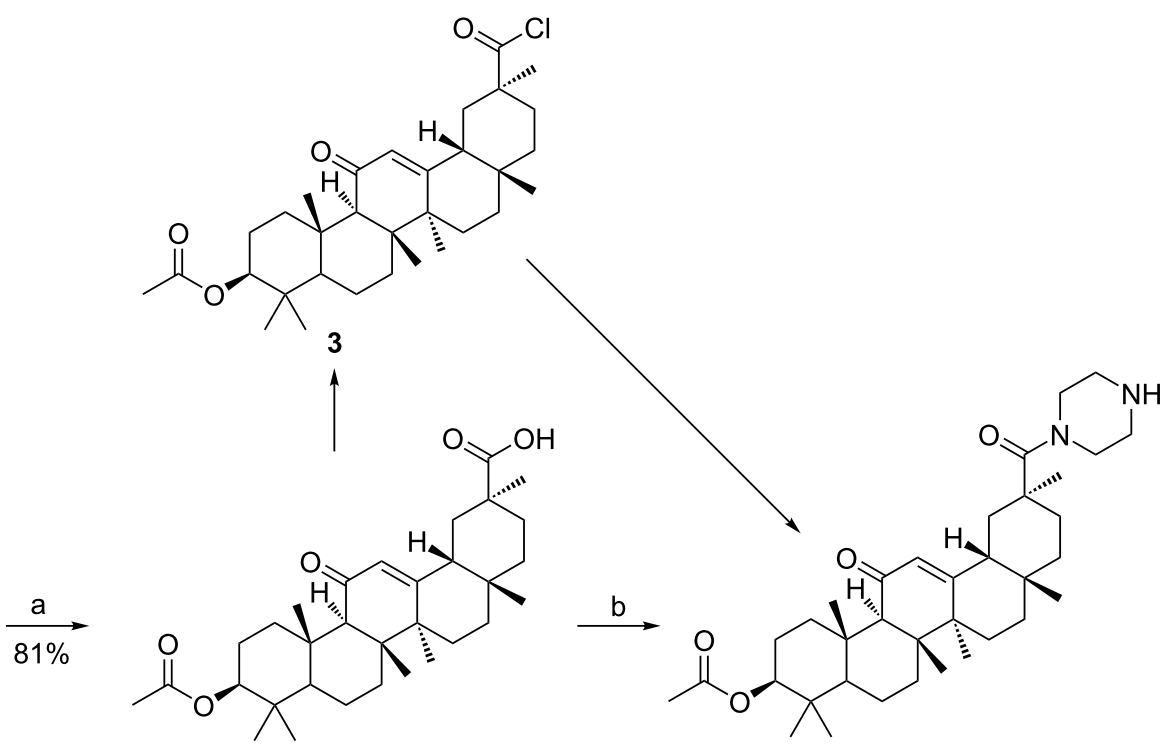

2

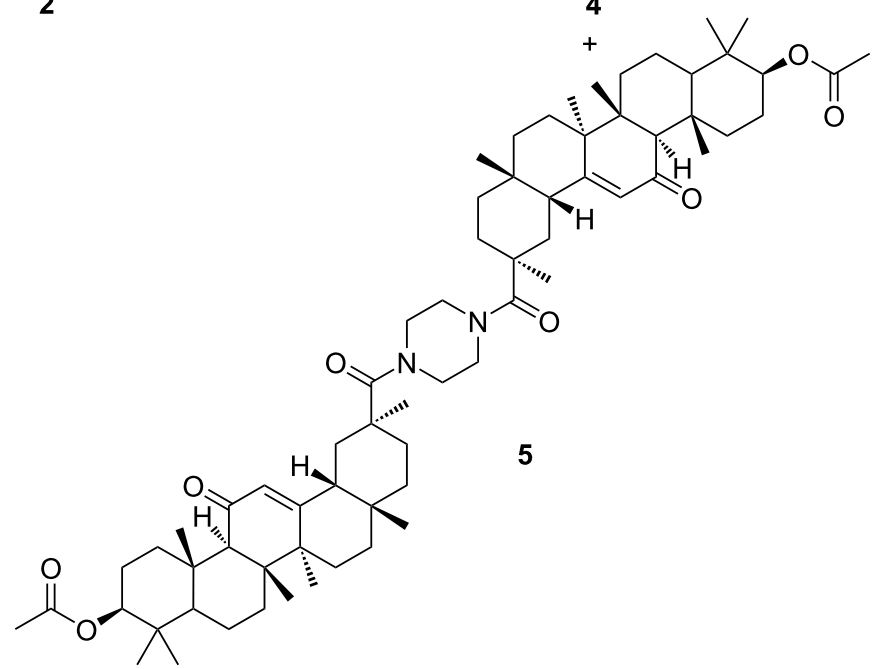


Table 1: Formation of piperazinyl amide 4: selected optimization reactions.

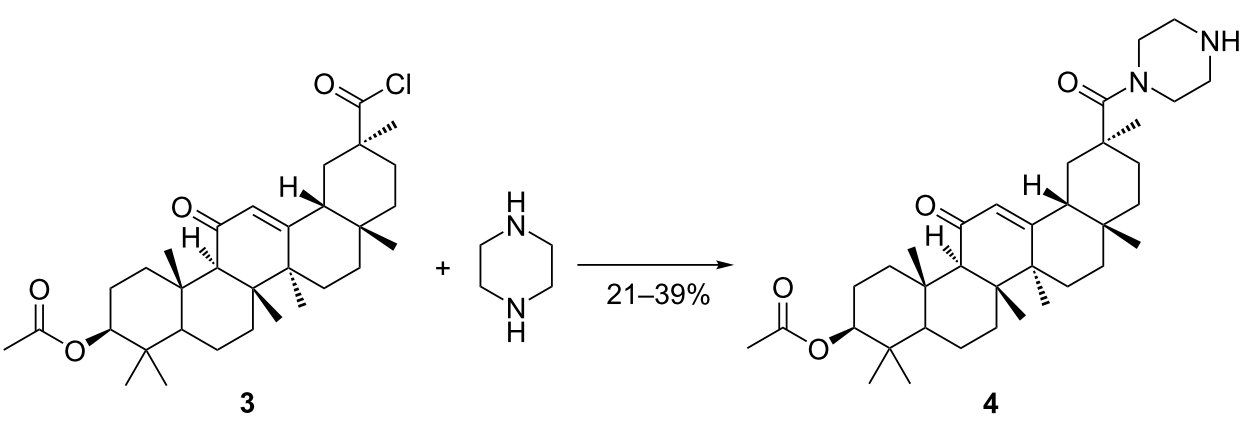

\begin{tabular}{|c|c|c|}
\hline entry & conditions $^{\mathrm{a}}$ & yield of $4^{b}$ \\
\hline 1 & piperazine ( 3 equiv), $0^{\circ} \mathrm{C}$ & $21 \%$ \\
\hline 2 & piperazine ( 6 equiv), $0^{\circ} \mathrm{C}$ & $28 \%$ \\
\hline 3 & piperazine (10 equiv), $0^{\circ} \mathrm{C}$ & $33 \%$ \\
\hline 4 & compound 3 (in $30 \mathrm{~mL}$ DCM) was added dropwise to the solution of piperazine (10 equiv), $0{ }^{\circ} \mathrm{C}$ & $36 \%$ \\
\hline 5 & compound 3 (in $100 \mathrm{~mL} \mathrm{DCM}$ ) was added dropwise to the solution of piperazine (10 equiv), $0^{\circ} \mathrm{C}$ & $39 \%$ \\
\hline 6 & compound 3 (in $100 \mathrm{~mL}$ DCM) was added dropwise to the solution of piperazine (10 equiv), $25^{\circ} \mathrm{C}$ & $32 \%$ \\
\hline
\end{tabular}

aReaction performed on a $0.90 \mathrm{mmol}$ scale of acyl chloride 3 . blsolated yield.

we decided to verify the feasibility of the feeding sequence in a model system. The solution with intermediate 3 was added dropwise to a solution of piperazine in $30 \mathrm{~mL}$ of dichloromethane (DCM). After the solutions were mixed, intermediate 3 was surrounded by piperazine molecules. The excess of piperazine could sufficiently avoid the formation of bisamide 5 (Table 1, entry 4). When intermediate 3 was dissolved in more DCM $(100 \mathrm{~mL})$, less side reactions occurred and the product was obtained in higher yield (Table 1, entries 4 and 5). By altering the amidation reaction temperature, the yield of amide 4 showed an obvious variation. A reaction temperature led to excessive bisamide 5 (Table 1, entry 6). The results of the experiments demonstrated that the side reaction was inevitable, and the amount of bisamide $\mathbf{5}$ produced was dependent on the reaction temperature and material ratio. The isolation and purification of product $\mathbf{4}$ were significantly more difficult, causing an unavoidable loss of target compound as well.

The observation described in Scheme 1 and Table 1 led us to consider a new approach for preparing the piperazinyl amide 4 (Scheme 2). Two general procedures for the synthesis of compound $\mathbf{8}$ are documented. One method involves acetylation of $18 \beta$-glycyrrhetinic acid, followed by amidation of the resulting ester $\mathbf{2}$ to give compound $\mathbf{8}$. Finally, the piperazinyl amide $\mathbf{4}$ was synthesized by of N-Boc deprotection.

Another procedure for the synthesis of compound $\mathbf{8}$ involves acylation of compound $\mathbf{9}$, which was prepared by the reactions of $18 \beta$-glycyrrhetinic acid with 1-Boc-piperazine under similar reaction conditions. Compound $\mathbf{8}$ was prepared in virtually quantitative yield from compound $\mathbf{9}$ with acetic anhydride without solvent at $130{ }^{\circ} \mathrm{C}$. The acetic acid formed in the reaction need not be neutralized and can escape from the reaction system. The reaction gave the optimal yield at $125-135{ }^{\circ} \mathrm{C}$. When the temperature was below $125^{\circ} \mathrm{C}$, compound $9 \mathrm{did}$ not reacted completely after $48 \mathrm{~h}$ (TLC monitoring).

The reaction from $\mathbf{2}$ to $\mathbf{8}$ proceeded via the intermediate $\mathbf{6}$, which can be isolated. The intermediate $\mathbf{6}$ did not react with 1-Boc-piperazine in low boiling point solvents even after an extended reaction time (up to $72 \mathrm{~h}$ ). Heating the reaction using conventional or microwave methods had no effect on the conversion of intermediate $\mathbf{6}$ to compound $\mathbf{8}$.

To explain the stable structure of the intermediate 6 (Figure 2a), energy minimization by MM2 was performed using the ChemBio3D Ultra 14.0 (CambridgeSoft Corporation, 2014) software force field [22]. The estimated structure was supported by crystal structures of similar structural compounds [23]. Figure $2 b$ suggests that the steric hindrance around the C30 ester group of intermediate $\mathbf{6}$ has a remarkable influence on the aminolysis reaction. The mechanism of the aminolysis reaction revealed that a higher reaction temperature of the solvent mixture contributed to the synthesis of compound $\mathbf{8}$.

To promote the yield of compound $\mathbf{8}$ and improve the overall yield of the reaction, an optimization of the reaction conditions was performed (Table 2). First, by using low boiling point sol- 


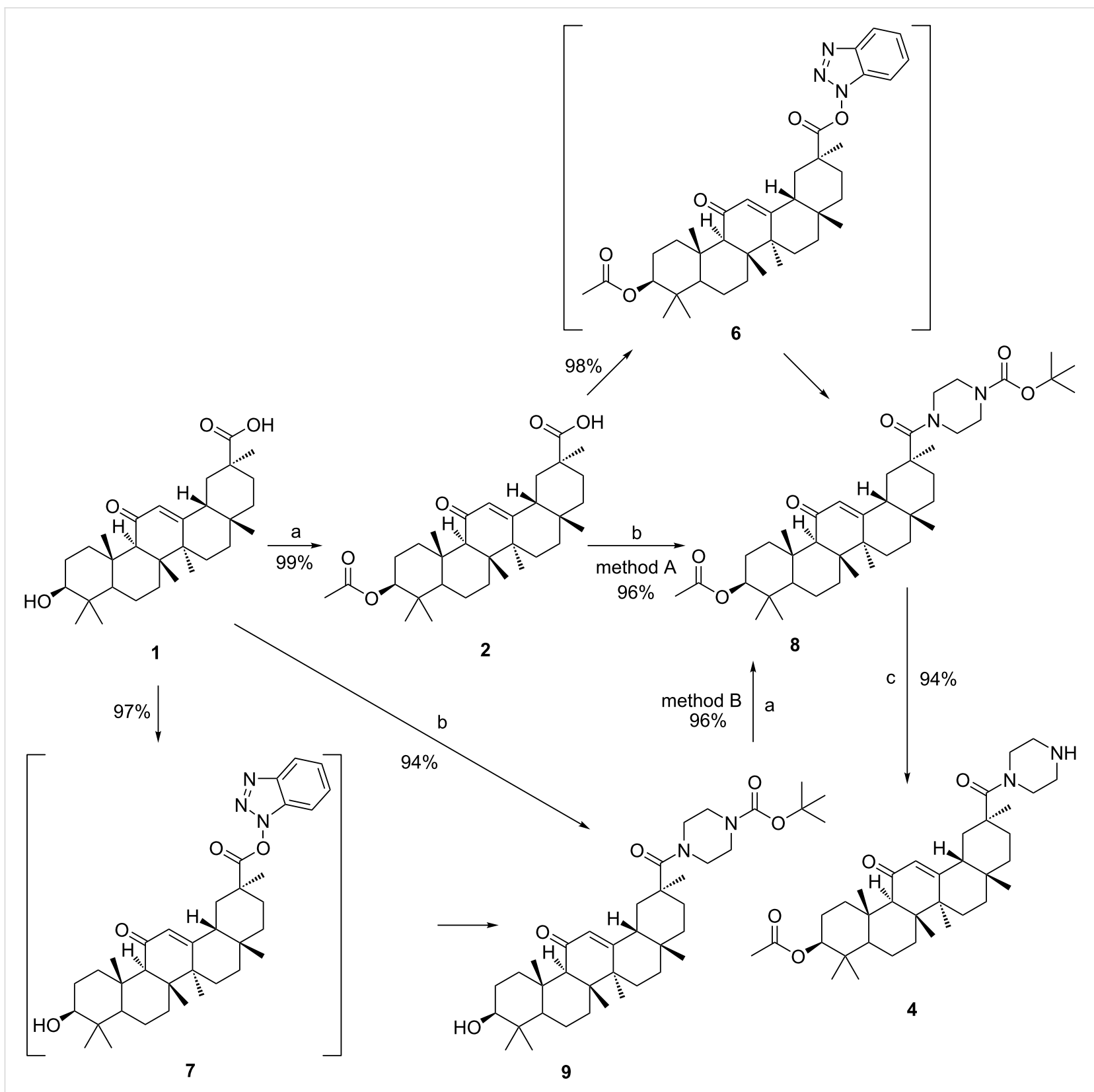

Scheme 2: Synthesis of compound 4. Reagents and conditions: (a) $\mathrm{Ac}_{2} \mathrm{O}, 130{ }^{\circ} \mathrm{C}, 1 \mathrm{~h}$; (b) 1-Boc-piperazine, $\mathrm{CH}_{3} \mathrm{CN}, \mathrm{NEt}$, $\mathrm{EDCl}, \mathrm{HOBt}$, reflux, $24 \mathrm{~h}$; (c) TFA, DCM, $0 / 25^{\circ} \mathrm{C}$.

a)

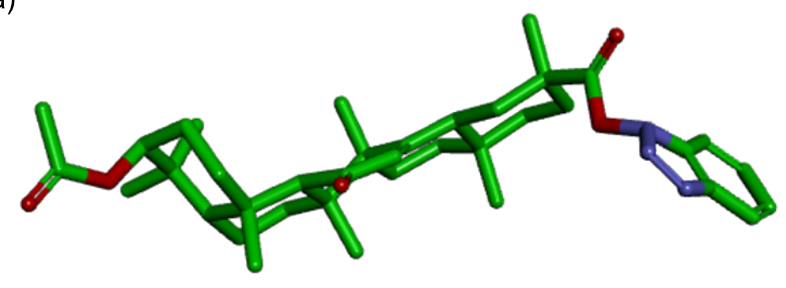

b)

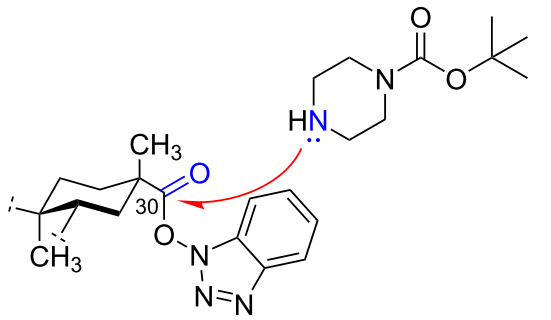

Figure 2: a) Estimated structure of the intermediate 6; b) Possible aminolysis process. 
vents, only low or no yields $(<19 \%)$ of the desired compound $\mathbf{8}$ were obtained after a longer reaction time (Table 2, entries 1-4). A further investigation of the reaction using microwave irradiation to heat at reflux temperature also failed to produce the target product (Table 2, entry 2). The intermediate 6 exhibited very low solubility in ethyl acetate solution, thereby directly causing the incomplete conversion of the substrate over several days (Table 2, entry 5). The reaction performed in acetonitrile as solvent achieved an excellent yield within $10 \mathrm{~h}$ but still with a small amount of intermediate 6 (Table 2, entry 6). Besides, $\mathrm{N}, \mathrm{N}$-dimethylformamide (DMF), a solvent with a higher boiling point than acetonitrile, was also tested, yet the reaction led to the formation of unknown compounds (TLC monitoring, Table 2, entry 7).

After screening several solvents, the optimal yield was obtained in acetonitrile, though a small amount of unconverted intermediate $\mathbf{6}$ was observed in the reaction mixture (Table 2, entry 6). Next, the reactivity of compound 2 with 1-Boc-piperazine in a 1:1.5 ratio was investigated. The reaction did not proceed completely in acetonitrile at reflux temperature, even after the period was prolonged to $48 \mathrm{~h}$. In contrast, 1-Boc-piperazine ( 2.5 equiv) exhibited high performance, thereby leading to the preparation of compound $\mathbf{8}$ in $95 \%$ yield (Table 2, entry 8 ), which essentially completed conversion of the intermediate 6 within $10 \mathrm{~h}$.

Alternatively, the aminolysis reaction of the isolated intermediate 6 with 1-Boc-piperazine (2.5 equiv) without any other additives can readily proceed in acetonitrile at reflux temperature in $95 \%$ yield.

Having demonstrated the effectiveness of the new procedure, we decided to apply this method towards the synthesis of compound 10. But our investigations proved that the above experimental conditions seem not to be suitable for the synthesis of compound 10. Interestingly, treatment of compound 9 with chloroacetic anhydride by heating did not afforded compound 10, but obtained another compound, 11 (Scheme 3).

In our analysis, compound $\mathbf{9}$ reacted with acetic anhydride to give compound $\mathbf{8}$ and acetic acid. At the reaction temperature, the acetic acid (boiling point $117-118{ }^{\circ} \mathrm{C}$ ) boiled and vapors escaped from the reaction mixture during the reaction process. However, in the reaction of compound 9 with chloroacetic anhydride (Scheme 3), the chloroacetic acid (boiling point $189^{\circ} \mathrm{C}$ )

Table 2: Optimization of the reaction conditions for the coupling of compound 2 with 1-Boc-piperazine.

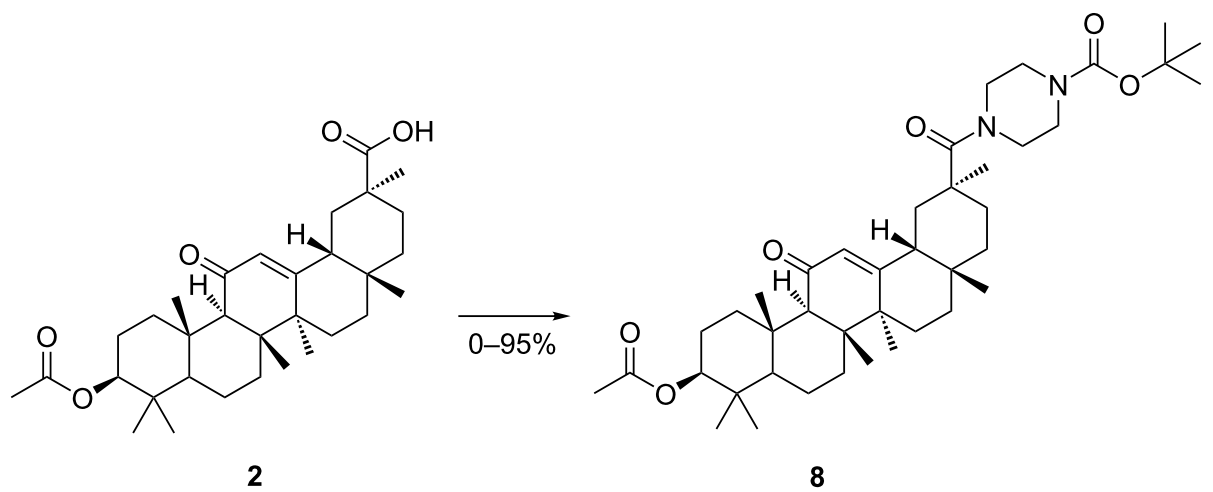

\begin{tabular}{|c|c|c|c|c|}
\hline entry & solvent & conditions $^{a}$ & yield of $6^{b}$ & yield of $8^{b}$ \\
\hline 1 & $\mathrm{CH}_{2} \mathrm{Cl}_{2}$ & reflux, $48 \mathrm{~h}$ & $93 \%$ & trace \\
\hline 2 & $\mathrm{CH}_{2} \mathrm{Cl}_{2}$ & reflux, 48 h, microwave & $93 \%$ & trace \\
\hline 3 & acetone & reflux, $48 \mathrm{~h}$ & $93 \%$ & trace \\
\hline 4 & THF & reflux, $48 \mathrm{~h}$ & $81 \%$ & $19 \%$ \\
\hline 5 & EtOAc & reflux, $48 \mathrm{~h}$ & $37 \%$ & $61 \%$ \\
\hline 6 & acetonitrile & reflux, $10 \mathrm{~h}$ & $12 \%$ & $88 \%$ \\
\hline 7 & DMF & reflux, $5 \mathrm{~h}, 130^{\circ} \mathrm{C}$ & 0 & 0 \\
\hline 8 & acetonitrile & $\begin{array}{l}\text { reflux, } 10 \mathrm{~h} \text {, } \\
\text { 1-Boc-piperazine }(2.5 \mathrm{mmol})\end{array}$ & 0 & $95 \%$ \\
\hline
\end{tabular}

a $2(1.0 \mathrm{mmol}), \mathrm{EDCl}(1.2 \mathrm{mmol}), \mathrm{NEt}_{3}(1.2 \mathrm{mmol}), \mathrm{HOBt}(1.2 \mathrm{mmol})$ and 1-Boc-piperazine $(1.2 \mathrm{mmol})$ were dissolved in $10 \mathrm{~mL}$ of solvent for the indicated time. ${ }^{\mathrm{b}}$ Isolated yield. 


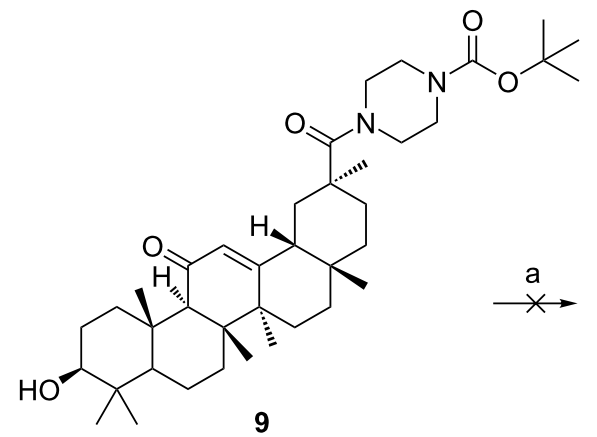

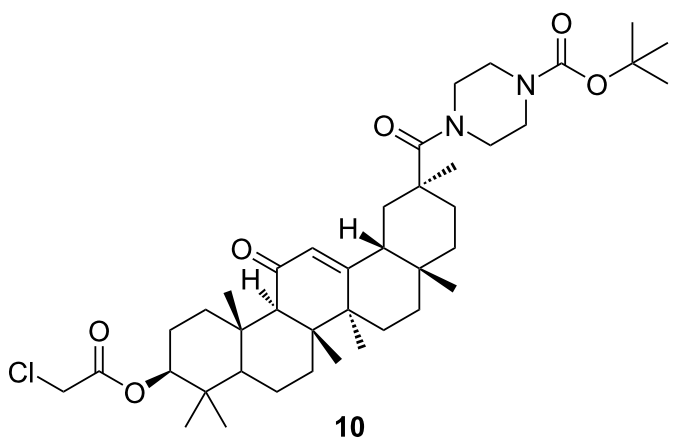

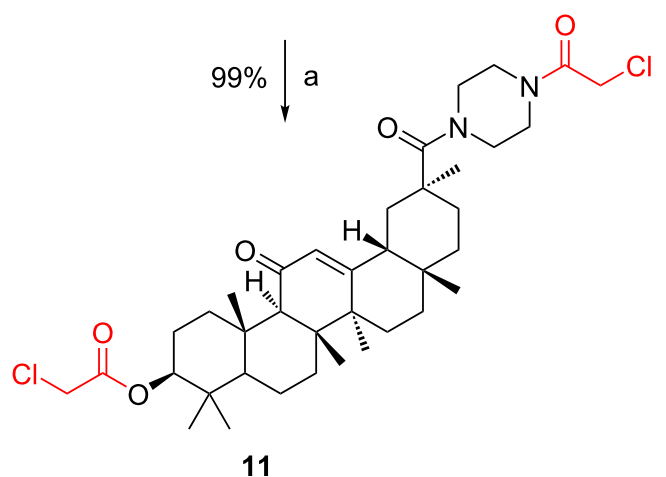

Scheme 3: Synthesis of byproduct 11 . Reagents and conditions: (a) chloroacetic anhydride, $130{ }^{\circ} \mathrm{C}, 1 \mathrm{~h}$.

formed during the reaction process cannot escape from the reaction system in the form of a gas, therefore, the initially formed compound 10 undergo $N$-Boc deprotection in an acidic medium to give byproduct 11. The structure of the byproduct $\mathbf{1 1}$ was confirmed by HRMS analysis.

Another noteworthy factor is that the acidity of chloroacetic acid $\left(\mathrm{p} K_{\mathrm{a}}=2.87\right)$ was stronger than that of acetic acid $\left(\mathrm{p} K_{\mathrm{a}}=4.76\right)$. The procedure for the generation of byproduct 11 was similar to $N$-Boc-deprotection using trifluoroacetic acid.

The effective synthesis of compound $\mathbf{1 0}$ was then explored (Table 3). In our initial attempt, compound 9 was reacted with chloroacetic anhydride (Table 3 , entry 1 ) at $130{ }^{\circ} \mathrm{C}$, whereas the reaction was complicated by the side formation of byproduct 11, which was obviously due to the presence of chloroacetic acid. Next, the reactions were performed with organic or inorganic bases to tie up or neutralize the chloroacetic acid released in the reaction. The base was provided in an amount or in a slight excess corresponding to the quantity required to tie up or neutralize the theoretical amount of acid released. The excess base did not affect the reaction and was washed off with water after the reaction. Triethylamine is a weak organic base that cannot completely neutralize the acid generated in the reaction. It may also volatilize at this reaction temperature (Table 3, entry 2). When the inorganic base was used as an acid-binding agent, the $\mathrm{H}_{2} \mathrm{O}$, produced during the neutralization reaction, consumed considerable quantities of chloroacetic anhydride (Table 3, entries 3 and 4). Thus, when the amount of chloroacetic anhydride was elevated to 20 equiv, compound 9 were not completely converted within $48 \mathrm{~h}$ under reflux conditions. Next, compound 9 was reacted with chloroacetic anhydride in refluxing toluene in the presence of $\mathrm{K}_{2} \mathrm{CO}_{3}$ in a Dean-Stark apparatus to remove the water formed during the reaction (Table 3, entry 5). Under such conditions, the reaction was completed in $1 \mathrm{~h}$ to give product $\mathbf{1 5}$, though a small amount of byproduct 11 was observed in the reaction mixture. Examples of suitable inorganic bases include $\mathrm{Na}_{2} \mathrm{CO}_{3}, \mathrm{NaHCO}_{3}$, and especially $\mathrm{K}_{2} \mathrm{CO}_{3}$. The synthesis of compound 9 was described in a similar manner in [11].

In another reaction, 18 $\beta$-glycyrrhetinic acid was smoothly esterified with chloroacetic anhydride at the $\mathrm{C} 3-\mathrm{OH}$ group to form compound 12 in a quantitative yield. The reaction was completed in $30 \mathrm{~min}$. The principal advantages of this method are that there is no solvent required, and that the reaction time is shorter than in previous cases [16], since the acid formed during the reaction may catalyze the esterification reaction of $18 \beta$ glycyrrhetinic acid with chloroacetic anhydride.

Having accessed compound 12, many other new compounds can also be prepared by replacing an amine with the halogen 


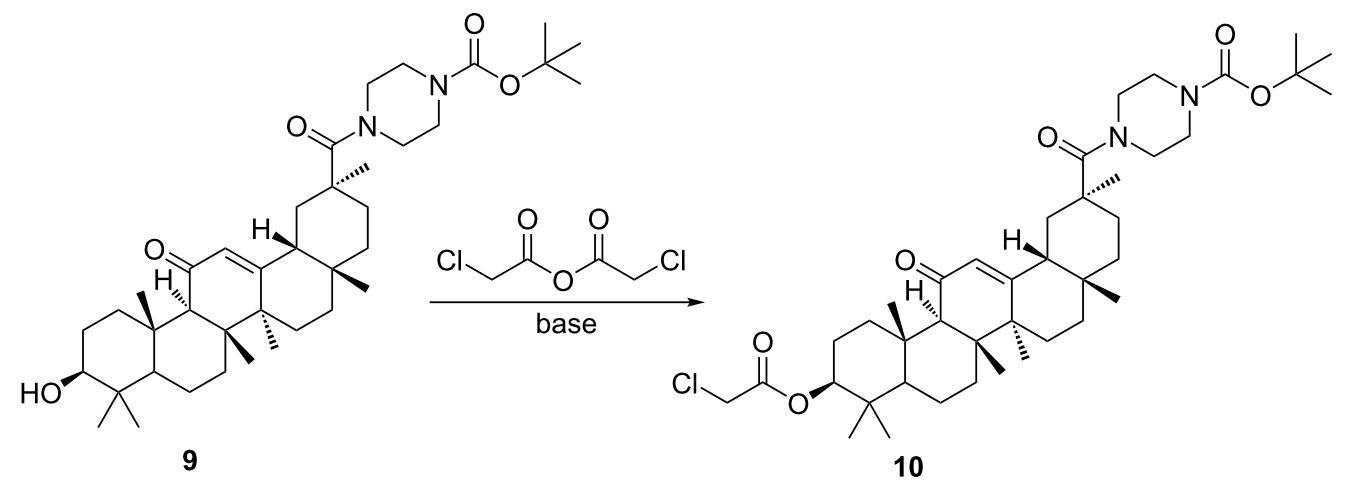

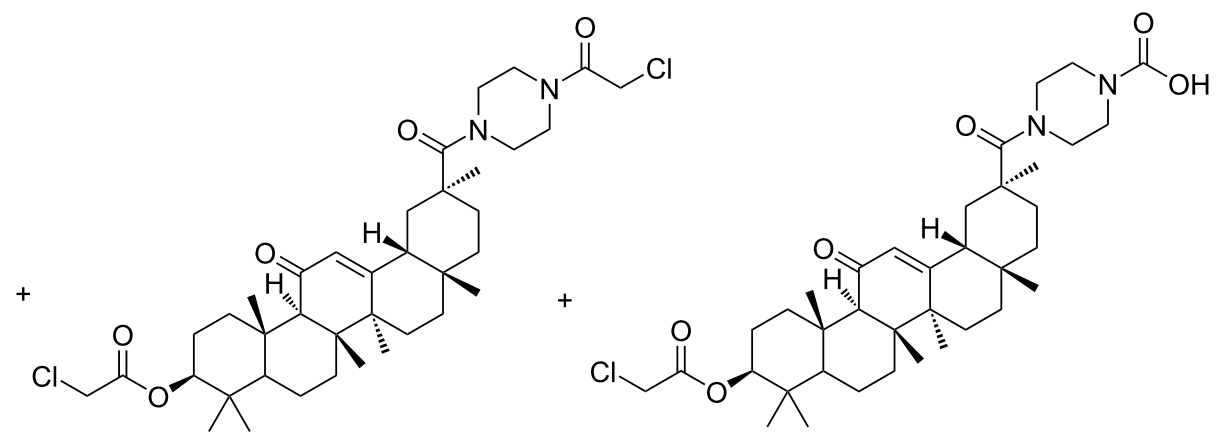

11

15

\begin{tabular}{|c|c|c|c|c|}
\hline entry & conditions $^{\mathrm{a}}$ & yield of $10^{b}$ & yield of $11^{b}$ & yield of $15^{b}$ \\
\hline 1 & chloroacetic anhydride (10 equiv) & 0 & 99 & 0 \\
\hline 2 & chloroacetic anhydride (10 equiv), $\mathrm{NEt}_{3}$, acetonitrile & 0 & 90 & trace \\
\hline 3 & chloroacetic anhydride (10 equiv), $\mathrm{K}_{2} \mathrm{CO}_{3}$, acetonitrile & 0 & 34 & trace \\
\hline 4 & chloroacetic anhydride (20 equiv), $\mathrm{K}_{2} \mathrm{CO}_{3}$, acetonitrile & 0 & 36 & trace \\
\hline 5 & chloroacetic anhydride (8 equiv), $\mathrm{K}_{2} \mathrm{CO}_{3}$, toluene, water separator & 0 & trace & 96 \\
\hline 6 & chloroacetic anhydride (8 equiv), $\mathrm{Na}_{2} \mathrm{CO}_{3}$, toluene, water separator & 0 & trace & 92 \\
\hline 7 & chloroacetic anhydride (8 equiv), $\mathrm{NaHCO}_{3}$, toluene, water separator & 0 & trace & 91 \\
\hline
\end{tabular}

aReaction performed on a $10 \mathrm{mmol}$ scale. blsolated yield.

atom. With compounds $\mathbf{1 2}$ and $\mathbf{1 5}$ in hand, we then tried to synthesize target compound $\mathbf{1 7}$ (Scheme 4), which is a novel $18 \beta$-glycyrrhetinic acid derivative to biologically active substances.

According to the first method, compound 12 reacted with morpholine in the presence of $\mathrm{K}_{2} \mathrm{CO}_{3} / \mathrm{I}_{2}$ to give compound $\mathbf{1 3}$, followed by successive amidation and $\mathrm{N}$-Boc deprotection gave compound 17. It is noteworthy that the carboxyl group of compound 12 did not react with inorganic bases, such as $\mathrm{K}_{2} \mathrm{CO}_{3}$, $\mathrm{Na}_{2} \mathrm{CO}_{3}, \mathrm{NaHCO}_{3}$, etc.

Another approach for the synthesis of compound $\mathbf{1 7}$ is the amination of compound $\mathbf{1 5}$ to give compound 16. Subsequently, compound 17 was obtained by hydrolysis of compound $\mathbf{1 6}$ using TFA in DCM. The synthesis of compounds 15-17 was described in a similar manner in [11].

After the $\mathrm{C} 3$ position has been converted to a stable ester structure, the piperazinyl amides of $18 \beta$-glycyrrhetinic acid can be also converted to novel unsymmetrical amides. For example, treatment of compound $\mathbf{4}$ with acyl chloride in dry DCM under alkaline conditions directly afforded $18 \beta$-glycyrrhetinic acid derivative 18. The molecular structure of compound $\mathbf{1 8}$ in the crystalline state is shown in Figure 3. In this crystal structure, there is an orientational disorder of the $m$-fluorophenyl moiety due to the rotation of a single bond. Details for the crystal structure determinations are given in Table 4. 


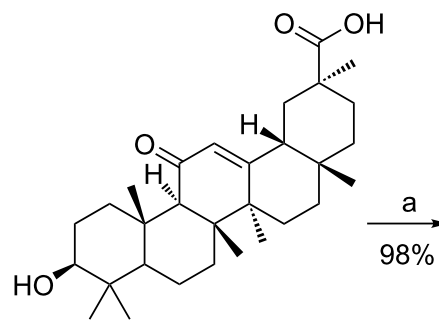

1<smiles>[3H][As](=O)(=O)O</smiles><smiles>CCN(C)C(=O)[C@]1(C)CC[C@@]2(C)CC[C@]3(C)C(=CC(=O)[C@]4(C)[C@@H]3CCC3C(C)(C)[C@@H](O)CC[C@@]34C)[C@]2(C)C1</smiles>

9

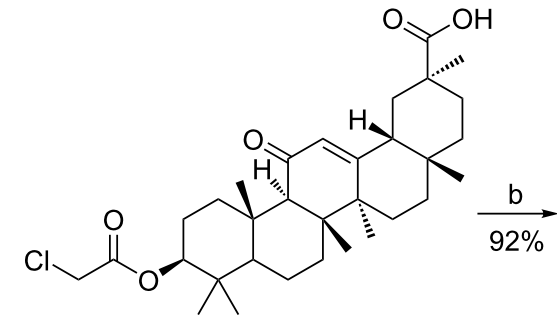

12

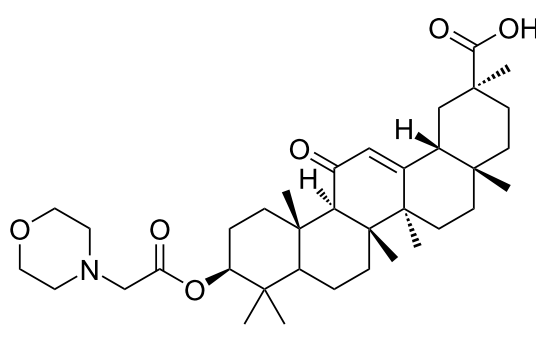

13

$\downarrow$<smiles>CCC(CC)[C@H](CC)OC(=O)CCl</smiles><smiles>Oc1ccc(O)cc1</smiles><smiles></smiles><smiles>[Y]C1[C@H](C)[C@@](C)(C=O)CC[C@H]1OC(=O)CN1CCOCC1</smiles>

14 $92 \% \downarrow d$

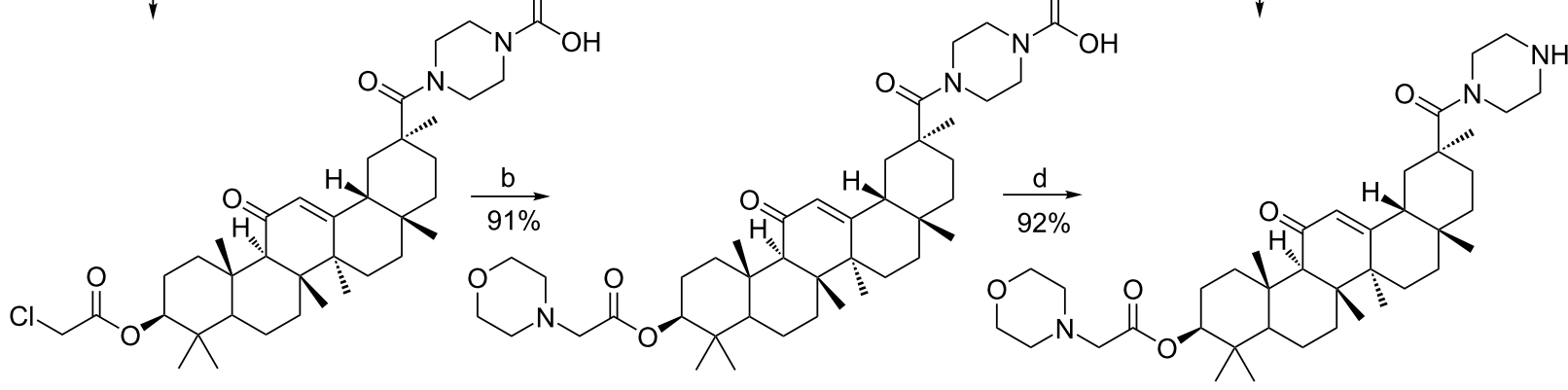
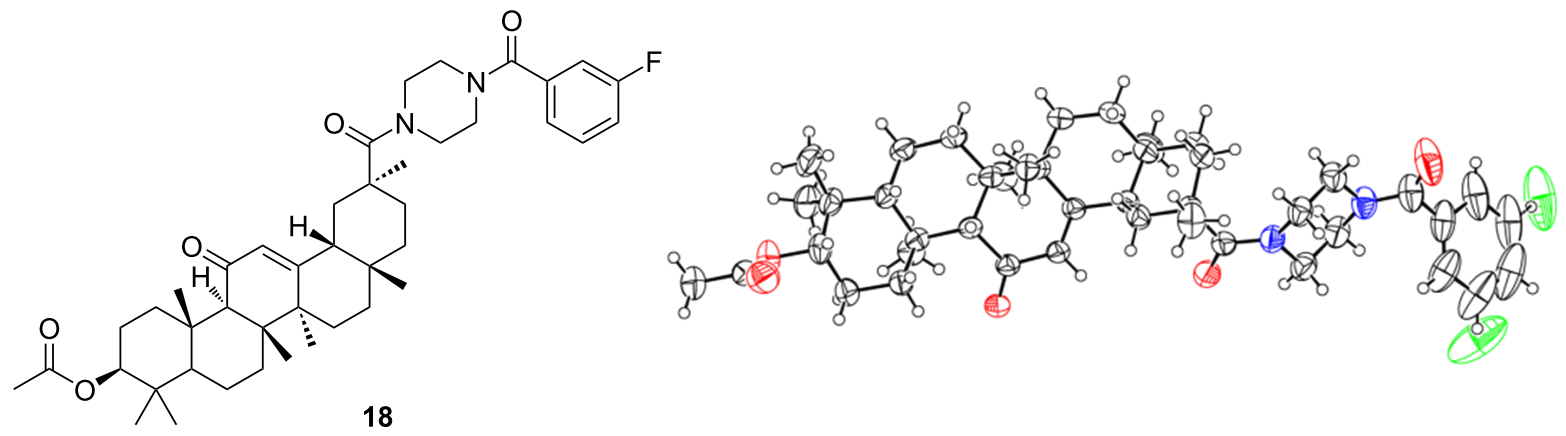

Figure 3: Crystal structure of conpound 18. 
Table 4: Crystallographic data of compound 18

$\begin{array}{ll}\text { CCDC number } & 1904891 \\ \text { formula } & \mathrm{C}_{43} \mathrm{H}_{59} \mathrm{FN}_{2} \mathrm{O}_{5} \\ \text { formula weight } & 702.92 \\ \text { crystal colour, shape } & \text { colourless } \\ \text { Solution } & \mathrm{CH}_{3} \mathrm{OH} \\ \text { crystal system } & \text { monoclinic } \\ \text { space group } & \mathrm{P} 2_{1} 2{ }_{1} 2_{1} \\ a / \AA & 11.581(3) \\ b / \AA & 12.216(3) \\ C / \AA & 27.749(7) \\ \alpha\left(^{\circ}\right) & 90 \\ \beta\left(^{\circ}\right) & 90 \\ Y\left(^{\circ}\right) & 90 \\ V / \AA^{3} & 3925.7(17) \\ T / \mathrm{K} & 273 \\ Z & 4 \\ \text { density (calculated) } / \mathrm{g} \cdot \mathrm{cm}^{-3} & 1.189 \\ F(000) & 1520 \\ R \text { (reflections) } & 0.0439(5028) \\ w R^{2} \text { (reflections) } & 0.1130(7118)\end{array}$

\section{Conclusion}

To sum up, piperazinyl amides of $18 \beta$-glycyrrhetinic acid were prepared from the $18 \beta$-glycyrrhetinic acid or its corresponding acyl chloride and piperazine. In this case, a considerable number of byproducts were inevitably produced. To reduce and/or avoid the formation of byproducts, efficient procedures were developed to prepare $18 \beta$-glycyrrhetinic acid derivatives that contain a terminal piperazinyl amide fragment. Due to the steric hindrance around the $\mathrm{C} 30$ ester group of intermediates, the intermediates $\mathbf{6}$ and $\mathbf{8}$ do not react with 1-Boc-piperazine at lower reaction temperature. The results showed the temperature dependence of the reaction between $18 \beta$-glycyrrhetinic acid and 1-Boc-piperazine. Besides, the Boc-piperazinyl amides of $18 \beta$ glycyrrhetinic acid reacted with chloroacetic anhydride in the presence of a base to give product $\mathbf{1 5}$. If no base is present, the Boc-piperazinyl amide of $18 \beta$-glycyrrhetinic acid 9 will undergo $N$-Boc deprotection and amidation to give compound 11. These results can be used to produce $N$-substituted piperazines of $18 \beta$-glycyrrhetinic acid in high yield, and further to develop more asymmetric piperazinamide derivatives. The structure of the asymmetric piperazinyl amide was confirmed by X-ray analysis.

\section{Experimental}

Detailed synthetic procedures for all new compounds including copies of their NMR spectra can be found in Supporting Information File 1.

\section{Supporting Information}

\section{Supporting Information File 1}

Preparation procedures and analytical data of compounds $\mathbf{1}$, 4-9, 11, 13, 15-18.

[https://www.beilstein-journals.org/bjoc/content/ supplementary/1860-5397-16-73-S1.pdf]

\section{Funding}

This research was funded by the Natural Science Foundation of the Liaoning Province (No. 20170540396 and No. 2019-ZD0693) and the Undergraduate innovation and entrepreneurship training program (No. 2019052).

\section{References}

1. Su, L.; Wang, Z.; Huang, F.; Lan, R.; Chen, X.; Han, D.; Zhang, L.; Zhang, W.; Hong, J. Environ. Toxicol. Pharmacol. 2018, 60, 82-90. doi:10.1016/j.etap.2018.04.012

2. Yano, S.; Harada, M.; Watanabe, K.; Nakamaru, K.; Hatakeyama, Y.; Shibata, S.; Takahashi, K.; Mori, T.; Hirabayashi, K.; Takeda, M.; Nagata, N. Chem. Pharm. Bull. 1989, 37, 2500-2504. doi:10.1248/cpb.37.2500

3. Agarwal, M. K.; Iqbal, M.; Athar, M. Redox Rep. 2005, 10, 151-157. doi:10.1179/135100005x57346

4. Jin, L.; Huang, R.; Huang, X.; Zhang, B.; Ji, M.; Wang, H. Bioorg. Med. Chem. 2018, 26, 1759-1775. doi:10.1016/j.bmc.2018.02.021

5. Kim, H. K.; Park, Y.; Kim, H. N.; Choi, B. H.; Jeong, H. G.; Lee, D. G.; Hahm, K.-S. Biotechnol. Lett. 2002, 24, 1899-1902. doi:10.1023/a:1020900124997

6. Jeong, H. G.; You, H. J.; Park, S. J.; Moon, A. R.; Chung, Y. C.; Kang, S. K.; Chun, H. K. Pharmacol. Res. 2002, 46, 221-227. doi:10.1016/s1043-6618(02)00121-4

7. Tolstikov, G. A.; Baltina, L. A.; Shul'Ts, E. E.; Pokrovskił, A. G. Bioorg. Khim. 1997, 23, 691-709.

8. Lallemand, B.; Gelbcke, M.; Dubois, J.; Prévost, M.; Jabin, I.; Kiss, R. Mini-Rev. Med. Chem. 2011, 11, 881-887. doi:10.2174/138955711796575443

9. Xu, B.; Wu, G.-R.; Zhang, X.-Y.; Yan, M.-M.; Zhao, R.; Xue, N.-N.; Fang, K.; Wang, H.; Chen, M.; Guo, W.-B.; Wang, P.-L.; Lei, H.-M. Molecules 2017, 22, 924. doi:10.3390/molecules22060924

10. Sharma, R.; Guru, S. K.; Jain, S. K.; Pathania, A. S.; Vishwakarma, R. A.; Bhushan, S.; Bharate, S. B. Med. Chem. Commun. 2015, 6, 564-575. doi:10.1039/c4md00344f

11. Dong, C.; YuQi, S.; DianShen, Y.; YiXia, G.; YunHong, J. The piperazine amide derivatives and the preparation method and application thereof of $18 \beta$-enoxolone. Chin. Patent CN201811582206.4A, Dec 24, 2018.

12. Yan, T.-L.; Bai, L.-F.; Zhu, H.-L.; Zhang, W.-M.; Lv, P.-C. ChemMedChem 2017, 12, 1087-1096. doi:10.1002/cmdc.201700271

13. Szakács, Z.; Béni, S.; Varga, Z.; Örfi, L.; Kéri, G.; Noszál, B. J. Med. Chem. 2005, 48, 249-255. doi:10.1021/jm049546c

14. Gang-Yue, L.; Sheng-Gang, Y.; Shan, J.; Xu-Hong, Q.; Qing-Chun, H.; Rong, Z. Chin. J. Org. Chem. 2008, 28, 2001-2006. 
15. Kowalski, P.; Kowalska, T.; Mokrosz, M.; Bojarski, A.; Charakchieva-Minol, S. Molecules 2001, 6, 784-795. doi:10.3390/60900784

16. Sommerwerk, S.; Heller, L.; Kerzig, C.; Kramell, A. E.; Csuk, R. Eur. J. Med. Chem. 2017, 127, 1-9. doi:10.1016/j.ejmech.2016.12.040

17. Kurono, M.; Unno, R.; Kimura, H.; Oka, M.; Hasegawa, K.; Ikeda, S.; Kuboyama, N.; Ito, T.; Sawai, K.; Ito, S. Glycyrrhetic acid derivatives and use thereof. U.S. Patent US4871740, Oct 3, 1989.

18. Chen, C.; Li, X.; Li, T.; Zhou, X. Acta Chim. Sin. (Chin. Ed.) 2012, 70, 852-858. doi:10.6023/a1111051

19. Couxi, C.; Mengxue, W.; Xueqiang, L.; Tiancai, L.; Xuezheng, Z. Chin. J. Org. Chem. 2015, 35, 835-842.

20. Lallemand, B.; Chaix, F.; Bury, M.; Bruyère, C.; Ghostin, J.; Becker, J.-P.; Delporte, C.; Gelbcke, M.; Mathieu, V.; Dubois, J.; Prévost, M.; Jabin, I.; Kiss, R. J. Med. Chem. 2011, 54, 6501-6513. doi:10.1021/jm200285z

21. Asakawa, C.; Ogawa, M.; Fujinaga, M.; Kumata, K.; Xie, L.; Yamasaki, T.; Yui, J.; Fukumura, T.; Zhang, M.-R. Bioorg. Med. Chem. Lett. 2012, 22, 3594-3597. doi:10.1016/j.bmcl.2012.04.049

22. Ikeda, H.; Ikuta, N.; Nakata, D.; Ishida, Y.; Terao, K. Bull. Chem. Soc. Jpn. 2015, 88, 1123-1127. doi:10.1246/bcsj.20140335

23. Beseda, I.; Czollner, L.; Shah, P. S.; Khunt, R.; Gaware, R.; Kosma, P.; Stanetty, C.; del Ruiz-Ruiz, M. C.; Amer, H.; Mereiter, K.; Cunha, T. D.; Odermatt, A.; Claßen-Houben, D.; Jordis, U. Bioorg. Med. Chem. 2010, 18, 433-454. doi:10.1016/j.bmc.2009.10.036

\section{License and Terms}

This is an Open Access article under the terms of the Creative Commons Attribution License (http://creativecommons.org/licenses/by/4.0). Please note that the reuse, redistribution and reproduction in particular requires that the authors and source are credited.

The license is subject to the Beilstein Journal of Organic Chemistry terms and conditions:

(https://www.beilstein-journals.org/bjoc)

The definitive version of this article is the electronic one which can be found at: $\underline{\text { doi:10.3762/bjoc. } 16.73}$ 